\title{
Traditional and Complementary Medicine Use in Patients With Type 2 Diabetes: Findings From A Multicenter Cross-Sectional Questionnaire Survey in Selangor, Malaysia
}

Article History

Received: 10 August 2021;

Received in Revised Form:

25 August 2021;

Accepted: 27 August 2021;

Available Online: 02

September 2021
Yieng Yii Wong', Azizul Hakim bin Sulaiman², Anis Aeida binti Mat Jaya $^{3}$, Pei Zan Wan ${ }^{4}$, Anchaya A/P Eh Wan ${ }^{5}$, Nurieshah Hanim binti Mohd Anuar ${ }^{3}$, Rajkumar A/L Selvaraju ${ }^{2}$, Kok Pim Kua ${ }^{1 *}$

${ }^{1}$ Puchong Health Clinic, Petaling District Health Office, Ministry of Health, 47100 Puchong, Selangor, Malaysia; wongyy2495@gmail.com

${ }^{2}$ Taman Medan Health Clinic, Petaling District Health Office, Ministry of Health, 46000 Petaling Jaya, Selangor, Malaysia; jijolakim@gmail.com

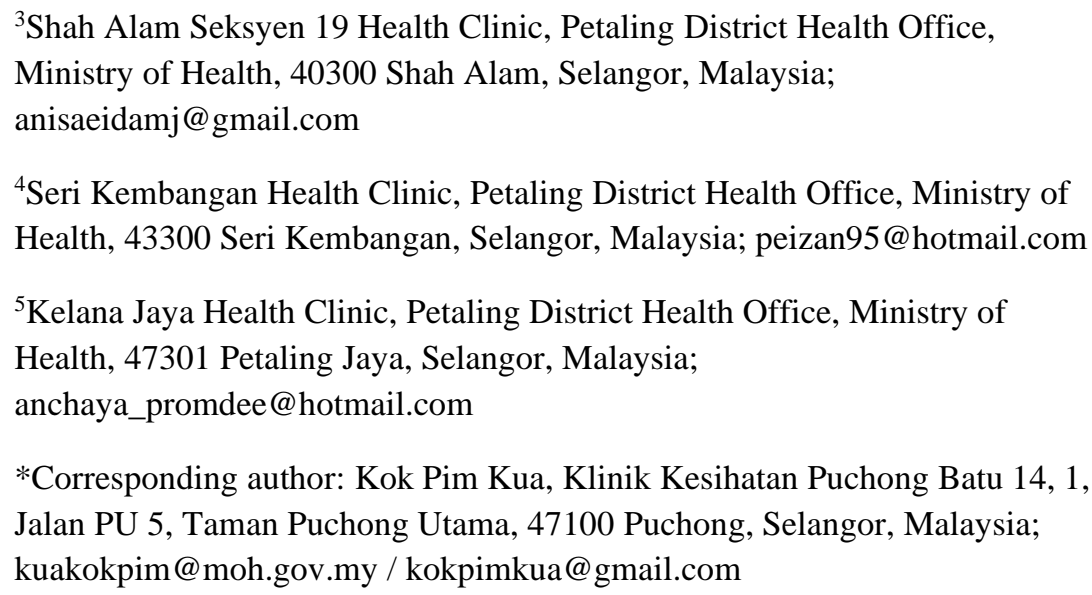

${ }^{4}$ Seri Kembangan Health Clinic, Petaling District Health Office, Ministry of Health, 43300 Seri Kembangan, Selangor, Malaysia; peizan95@ hotmail.com

${ }^{5}$ Kelana Jaya Health Clinic, Petaling District Health Office, Ministry of Health, 47301 Petaling Jaya, Selangor, Malaysia;

anchaya_promdee@hotmail.com

*Corresponding author: Kok Pim Kua, Klinik Kesihatan Puchong Batu 14, 1, Jalan PU 5, Taman Puchong Utama, 47100 Puchong, Selangor, Malaysia; kuakokpim@moh.gov.my / kokpimkua@gmail.com

Abstract: Current global estimates of traditional and complementary medicine (T\&CM) utilization range from $9.8 \%$ to $76 \%$, with high rates of use being documented in Japan, South Korea, and Malaysia. Previous research has shown that patients with diabetes are more likely to use T\&CM than individuals without diabetes. A multicenter cross-sectional survey of patients with type 2 diabetes mellitus was conducted at five primary care health clinics in Petaling district of Selangor, Malaysia from June 1, 2020 through September 30, 2020. A total of 476 patients were enrolled. $58.0 \%$ of men and $64.8 \%$ of women reported having used at least one type of T\&CM. The prevalence of T\&CM use among Malays, Chinese, and Indians was $71.1 \%, 33.3 \%$, and $62.9 \%$ respectively. The most commonly used T\&CM were herbal remedies $(45.2 \%)$, followed by nutritional supplements $(9.9 \%) .80 .0 \%$ of the patients would follow their doctor's instructions if the doctor asked them not to use T\&CM, whereas $70.2 \%$ would consult their doctor about using T\&CM. Factors associated with higher tendency for T\&CM use included increased age, unemployment, oral antidiabetic monotherapy, presence of family history of diabetes, and coexisting chronic disease. T\&CM therapies were common among the multi-ethnic patient population with type 2 diabetes. The high rate of use warranted clinical attention and intervention to prevent adverse drug events. 
Keywords: Traditional and complementary medicine, Complementary and alternative medicine, Diabetes, Drug-herb interaction, Dietary supplement, Prescription medication.

\section{Introduction}

Traditional and complementary medicine (T\&CM) refers to a wide variety of clinical therapies that are not currently considered as an integral part of conventional medicine ${ }^{[1]}$. The prevalence of T\&CM use by populations across the world ranges from $9.8 \%$ to $76 \%$, with high rates being documented in Japan (76\%), South Korea (75\%), and Malaysia $(30 \%)^{[2,3]}$. However, previous studies have revealed that about half of T\&CM users do not disclose their use of T\&CM with their primary care providers. This problem is compounded by the issue that, very often, physicians rarely initiate conversations or discuss with patients about T\&CM. A better understanding of practices, attitudes, and beliefs of patients towards T\&CM can facilitate better coordination of care, decrease the risk of potential interactions between prescription medications and T\&CM therapies, and lead to better patient outcomes ${ }^{[4]}$.

Diabetes mellitus is one of the most widespread and morbid chronic diseases worldwide and over $90 \%$ of the diagnoses are type $2^{[5]}$. While conventional medical therapies prevent some of its devastating complications, patients do not usually restore normoglycemia or eliminate all the adverse consequences. Hence, it is not surprising that individuals with diabetes are more likely to utilize T\&CM in conjunction with standard medical care than those without diabetes ${ }^{[6]}$. Although several T\&CM therapies have been suggested to have promising roles in diabetes, their safety concerns, clinical efficacy, and mechanism of action are still less understood. Patients with diabetes are often prescribed with multiple medications. Thus, there exists a substantial risk for clinically significant drug-herb and drugsupplement interactions ${ }^{[1]}$.

Sociocultural factors have been reported to influence the utilization of $\mathrm{T}_{\mathrm{C}} \mathrm{CM}^{[7]}$. There have been limited studies to elucidate the prevalence, knowledge, attitude, and pattern of T\&CM use among the multi-ethnic population in Malaysia. Consequently, we conducted a multicenter cross-sectional study to better understand the use of T\&CM among patients with type 2 diabetes.

\section{Materials and Methods}

\subsection{Study Population}

From June 1, 2020 until September 30, 2020, a cross-sectional questionnaire survey was administered via face-to-face interviews to patients with type 2 diabetes of 18 years of age or older who were receiving medical care at five primary care health clinics situated in 
the district of Petaling, Selangor, Malaysia. Potential participants were recruited using convenience sampling, i.e., non-random (non-probability) sampling when they were waiting or collecting their medications at the pharmacy unit. All participants who agreed to participate in the research had to provide a written informed consent. The survey took approximately 10 minutes to complete. The exclusion criteria included gestational diabetes, type 1 diabetes, and inability to comprehend the survey.

\subsection{Survey Instrument}

The questionnaire was developed by our team in dual language (English and Malay) and consisted of 25 questions to glean sociodemographic information, clinical characteristics, knowledge, attitude, and perception towards T\&CM, type of T\&CM used, and reasons for use of T\&CM. It was adopted from two validated questionnaire tools in previous studies ${ }^{[8,9]}$. The questions were further reviewed by four pharmacists for clarity and ease of administration.

\subsection{Sample Size}

According to the Malaysian National Health and Morbidity Survey 2019, the estimated adult population with known diabetes in the state of Selangor was $408,113^{[10]}$. Using the Raosoft calculator, the sample size of the study was enumerated based upon a margin of error of $5 \%$, a confidence level of $95 \%$, a response distribution of $50 \%$, and a total population of diabetic patients of approximately $408,113^{[11]}$. The recommended sample size was 384 patients.

\subsection{Statistical Analysis}

Data were summarized by the calculation of frequency and percentage for categorical variables. Chi-square tests were carried out to assess sociodemographic and clinical predictors of T\&CM use. Additionally, multivariable logistic regression analyses were performed to compute the odds ratio (OR) and 95\% confidence intervals (CI) for the use of T\&CM among patients with type 2 diabetes. A $\mathrm{P}$ value of less than 0.05 was considered to indicate statistical significance. All analyses were undertaken with the use of SPSS Statistics software, version 26 (IBM Corp., Armonk, NY, USA).

\subsection{Ethics Statement}

The research was conducted in accordance with the principles of the Malaysian Guideline for Good Clinical Practice, following the International Council for Harmonization of Good Clinical Practice Guidance. All study subjects gave written informed consent. The 
study protocol was approved by the Medical Research and Ethics Committee, Ministry of Health Malaysia (KKM/NIHSEC/ P20-984 [6]).

\section{Results}

476 patients participated in the study. A total of 123 of 212 men $(58.0 \%)$ reported having used at least one type of T\&CM, as compared with 171 of 264 women $(64.8 \%)$. Nearly three quarters of all T\&CM users were aged 51 years and older. The prevalence of T\&CM use among Malays, Chinese, and Indians was 71.1\%, 33.3\%, and 62.9\% respectively. Of 294 patients with type 2 diabetes reported using T\&CM, the majority attained secondary education (56.5\%), were married (94.2\%), or were non-smokers $(73.5 \%)$. In terms of clinical characteristics, most of the T\&CM users had a family history of diabetes $(74.5 \%)$, received oral antidiabetic monotherapy (51.7\%), had no diabetic complications (70.7\%), and had coexisting hypertension $(74.8 \%)$ or dyslipidemia $(65.0 \%)$. Further details are presented in Table 1.

More than one quarter of patients believed T\&CM was safe and effective for diabetes. $80.0 \%$ would follow their doctor's instructions if the doctor asked them not to use it, whereas $70.2 \%$ would discuss T\&CM use with their primary care physician before taking it. The main reasons for T\&CM use were belief in its efficacy in diabetes control (41.8\%) and wide availability with low costs $(10.1 \%)$. Herbal remedies were the most frequently used T\&CM (45.2\%) and the varieties reported encompassed finished products such as Momordica charantia (bitter melon), Trigonella foenum-graecum (fenugreek), Swietenia macrophylla (sky fruit), green apple herb, Allium sativum (garlic), Azadirachta indica (neem), Ayurvedic polyherbal formulation, Orthosiphon stamineus Benth (cat's whiskers), and Kaempferia parviflora (black ginger); and raw herbs such as Panex ginseng (Asian or Korean ginseng), Panex quinquefolius (American ginseng), Kasini veppalai, and Andrographis paniculata. Other T\&CM practices used by the patients included nutritional supplements (9.9\%), treatment based on a specific diet $(2.5 \%)$, wet cupping $(1.7 \%)$, honeybee products $(1.7 \%)$, Roqia (1.3\%), medical massage $(1.1 \%)$, and acupuncture $(0.8 \%)$.

Study participants were largely influenced by their friends (33.0\%) and family members $(18.7 \%)$ when making a decision to use T\&CM. A third of them were satisfied with current T\&CM and would continue using it for a long period of time (Table 2). Chi-square analysis showed that T\&CM use in type 2 diabetes was significantly associated with ethnicity, employment status, smoking status, coexisting chronic conditions, duration of diabetes diagnosis (All $\mathrm{P}<0.001$ ), presence of a diabetes complication $(\mathrm{P}=0.002)$, educational attainment $(\mathrm{P}=0.002)$, pharmacotherapy prescribed $(\mathrm{P}=0.003)$, and age $(\mathrm{P}=0.014)$ (Table 1$)$. 
Multivariate logistic regression analysis depicted elevated rates of T\&CM usage among age groups of 31 to 60 years, unemployed, oral antidiabetic monotherapy, presence of family history of diabetes, and coexisting illness (Table 3).

Table 1. Sociodemographic and clinical characteristics of patients in the study and T\&CM use ( $n=476)$.

\begin{tabular}{|c|c|c|c|}
\hline Characteristic & $\begin{array}{c}\text { Total number of } \\
\text { study participants, } \\
n(\%)\end{array}$ & $\begin{array}{c}\text { Number of patients } \\
\text { reported having used } \\
\text { T\&CM for diabetes, } n \text { (\%) }\end{array}$ & $\begin{array}{l}P \text { value } \\
\text { (Chi- } \\
\text { square } \\
\text { test) } \\
\end{array}$ \\
\hline \multicolumn{4}{|l|}{ Age (years) } \\
\hline$<30$ & $14(2.9)$ & $9(1.9)$ & \multirow{5}{*}{0.014} \\
\hline $31-40$ & $31(6.5)$ & $25(5.3)$ & \\
\hline $41-50$ & $80(16.8)$ & 49 (10.3) & \\
\hline $51-60$ & $156(32.8)$ & $106(22.3)$ & \\
\hline$>60$ & $195(41.0)$ & $105(22.1)$ & \\
\hline \multicolumn{4}{|l|}{ Gender } \\
\hline Male & $212(44.5)$ & $123(25.8)$ & \multirow{2}{*}{0.132} \\
\hline Female & $264(55.5)$ & $171(35.9)$ & \\
\hline \multicolumn{4}{|l|}{ Marital status } \\
\hline Single & $22(4.6)$ & $11(2.3)$ & \multirow{3}{*}{0.223} \\
\hline Married & 447 (93.9) & $277(58.2)$ & \\
\hline Other & $7(1.5)$ & $6(1.3)$ & \\
\hline \multicolumn{4}{|l|}{ Ethnicity } \\
\hline Malay & $235(49.4)$ & $167(35.1)$ & \multirow{4}{*}{$<0.001$} \\
\hline Chinese & $87(18.3)$ & $29(6.1)$ & \\
\hline Indian & $151(31.7)$ & $95(20.0)$ & \\
\hline Other & $3(0.6)$ & $3(0.6)$ & \\
\hline \multicolumn{4}{|l|}{ Educational level } \\
\hline Primary & $99(20.8)$ & $48(10.1)$ & \multirow{5}{*}{0.002} \\
\hline Secondary & $271(56.9)$ & $166(34.9)$ & \\
\hline Diploma & $66(13.9)$ & $48(10.1)$ & \\
\hline Undergraduate degree & $36(7.6)$ & $28(5.9)$ & \\
\hline Postgraduate degree & $4(0.8)$ & $4(0.8)$ & \\
\hline \multicolumn{4}{|l|}{ Employment status } \\
\hline Employed & $213(44.7)$ & $125(26.3)$ & \multirow{3}{*}{$<0.001$} \\
\hline Unemployed & $82(17.2)$ & $69(14.5)$ & \\
\hline Retired & $181(38.0)$ & $100(21.0)$ & \\
\hline \multicolumn{4}{|l|}{ Smoking status } \\
\hline Daily smoker & $72(15.1)$ & $30(6.3)$ & \multirow{4}{*}{$<0.001$} \\
\hline Non-daily smoker & $45(9.5)$ & $34(7.1)$ & \\
\hline Former smoker & $26(5.5)$ & $14(2.9)$ & \\
\hline Non-smoker & $333(70.0)$ & $216(45.4)$ & \\
\hline \multicolumn{4}{|l|}{$\begin{array}{l}\text { Duration of diagnosed type } 2 \\
\text { diabetes }\end{array}$} \\
\hline Newly diagnosed & $41(8.6)$ & $26(5.5)$ & \multirow{5}{*}{$<0.001$} \\
\hline$<5$ years & $114(23.9)$ & $82(17.2)$ & \\
\hline $5-10$ years & $161(33.8)$ & $94(19.7)$ & \\
\hline $10-15$ years & $109(22.9)$ & $52(10.9)$ & \\
\hline$>15$ years & $51(10.7)$ & $40(8.4)$ & \\
\hline \multicolumn{4}{|l|}{ Family history of diabetes } \\
\hline Yes & $351(73.7)$ & $219(46.0)$ & \multirow{3}{*}{0.125} \\
\hline No & 109 (22.9) & $69(14.5)$ & \\
\hline Unsure & $16(3.4)$ & $6(1.3)$ & \\
\hline \multicolumn{4}{|l|}{ Diabetes pharmacotherapy } \\
\hline Oral antidiabetic drugs alone & $217(45.6)$ & $152(31.9)$ & \multirow{2}{*}{0.003} \\
\hline Insulin alone & $53(11.1)$ & $29(6.1)$ & \\
\hline
\end{tabular}




\begin{tabular}{lccc}
\hline \multicolumn{1}{c}{ Characteristic } & $\begin{array}{c}\text { Total number of } \\
\text { study participants, } \\
\boldsymbol{n}(\boldsymbol{\%})\end{array}$ & $\begin{array}{c}\text { Number of patients } \\
\text { reported having used } \\
\text { T\&CM for diabetes, } \boldsymbol{n} \text { (\%) }\end{array}$ & $\begin{array}{c}\text { P value } \\
\text { (Chi- } \\
\text { square } \\
\text { test) }\end{array}$ \\
\hline Combination of oral hypoglycemic & $206(43.3)$ & $113(23.7)$ & \\
drugs and insulin & & & \\
Diabetes complications & $97(20.4)$ & $60(12.6)$ & \\
Neuropathy & $36(7.6)$ & $17(3.6)$ & \\
Cardiovascular complications & $47(9.9)$ & $16(3.4)$ & \\
Retinopathy & $12(0.2)$ & $1(0.2)$ & \\
Diabetic foot & $26(2.5)$ & $5(1.1)$ & \\
Nephropathy & $45(9.5)$ & $12(2.5)$ & \\
Multiple complications & $311(65.3)$ & $208(43.7)$ & \\
No complications & $357(75.0)$ & $220(46.2)$ & \\
Concomitant chronic disease & $290(60.9)$ & $191(40.1)$ & \\
Hypertension & $20(4.2)$ & $7(1.5)$ & \\
Dyslipidemia & $60(12.6)$ & $22(4.6)$ & \\
Asthma & $11(2.3)$ & $10(2.1)$ & \\
Coronary heart disease & $256(53.8)$ & $146(30.7)$ & \\
Other & $27(5.7)$ & $10(2.1)$ & \\
Multiple coexisting diseases & & & \\
No coexisting disease & & & \\
\hline
\end{tabular}

Table 2. Knowledge, attitude, and pattern of T\&CM use ( $n=476)$.

\begin{tabular}{lc}
\hline \multicolumn{1}{c}{ Variable } & Total number of patients, $\boldsymbol{n}(\boldsymbol{\%})$ \\
\hline Knowledge & \\
Hear of T\&CM & $373(78.4)$ \\
Believe T\&CM is effective for diabetes & $167(35.1)$ \\
Believe T\&CM is safe & $141(29.6)$ \\
Attitude & \\
Follow a doctor's instructions if the doctor asks you not to use T\&CM & $381(80.0)$ \\
Discuss with a doctor if you want to use T\&CM & $334(70.2)$ \\
Combine T\&CM with antidiabetic medication & $263(55.3)$ \\
Advise or encourage family member(s) to use T\&CM & $152(31.9)$ \\
Reason to use T\&CM & \\
Lack of confidence towards prescription medication & $18(3.8)$ \\
Long appointment intervals to see a doctor & $12(2.5)$ \\
Believe T\&CM has fewer side effects than conventional medicine & $47(9.9)$ \\
Believe T\&CM helps in diabetes control & $199(41.8)$ \\
Poor physician-patient communication & $4(0.8)$ \\
T\&CM is easily available and cheaper & $48(10.1)$ \\
Doctor suggests to use T\&CM & $27(5.7)$ \\
Other & $21(4.4)$ \\
Multiple reasons & $68(14.3)$ \\
Type of T\&CM & \\
Herbal remedies & $215(45.2)$ \\
Wet cupping & $8(1.7)$ \\
Nutritional supplements & $47(9.9)$ \\
Cautery & $0(0.0)$ \\
Roqia & $6(1.3)$ \\
Honeybee products & $8(1.7)$ \\
Medical massage & $5(1.1)$ \\
Treatment based on a specific diet & $12(2.5)$ \\
Acupuncture & $4(0.8)$ \\
Other & $27(5.7)$ \\
Multiple types of T\&CM & $26(5.5)$ \\
\hline & \\
\hline
\end{tabular}




\begin{tabular}{lc}
\hline \multicolumn{1}{c}{ Variable } & Total number of patients, $\boldsymbol{n}(\boldsymbol{\%})$ \\
\hline Influencer of T\&CM use & $157(33.0)$ \\
Friend & $8(1.7)$ \\
Traditional healer & $9(1.9)$ \\
Pharmacist & $5(1.1)$ \\
Herbalist & $4(0.8)$ \\
Dietitian & $9(1.9)$ \\
Physician & $89(18.7)$ \\
Family member & $31(6.5)$ \\
Other & $2(0.4)$ \\
Neighbor & $2(0.4)$ \\
Salesman & $16(3.4)$ \\
Online advertisement, TV, YouTube, or newspaper & $32(6.7)$ \\
Multiple sources of influence & \\
Perception & $153(32.1)$ \\
Satisfied with current T\&CM used & $157(33.0)$ \\
Will continue using T\&CM for the long term & $74(15.5)$ \\
Ever used T\&CM for medical conditions other than diabetes & \\
\hline
\end{tabular}

Table 3. Multivariate adjusted association between patients' characteristics and T\&CM use for type 2 diabetes $(n=476)$.

\begin{tabular}{|c|c|c|}
\hline \multirow{2}{*}{ Characteristic } & \multicolumn{2}{|c|}{ T\&CM use for type 2 diabetes } \\
\hline & OR $(95 \% \mathrm{CI})$ & $P$ value \\
\hline \multicolumn{3}{|l|}{ Age (years) } \\
\hline$<30$ & $1.983(0.504-7.805)$ & 0.327 \\
\hline $31-40$ & $6.864(2.105-22.380)$ & 0.001 \\
\hline $41-50$ & $3.255(1.601-6.616)$ & 0.001 \\
\hline $51-60$ & $3.403(1.848-6.264)$ & 0.000 \\
\hline$>60$ & 1 (ref) & - \\
\hline \multicolumn{3}{|l|}{ Gender } \\
\hline Male & $1.251(0.775-2.019)$ & 0.359 \\
\hline Female & $1(\mathrm{ref})$ & - \\
\hline \multicolumn{3}{|l|}{ Marital status } \\
\hline Single & $0.081(0.007-0.894)$ & 0.040 \\
\hline Married & $0.227(0.026-2.013)$ & 0.183 \\
\hline Other & $1($ ref $)$ & - \\
\hline \multicolumn{3}{|l|}{ Employment status } \\
\hline Employed & $0.468(0.263-0.833)$ & 0.010 \\
\hline Unemployed & $2.950(1.370-6.350)$ & 0.006 \\
\hline Retired & $1($ ref $)$ & - \\
\hline \multicolumn{3}{|l|}{ Smoking status } \\
\hline Daily smoker & $0.617(0.220-1.729)$ & 0.359 \\
\hline Non-daily smoker & $2.922(0.863-9.888)$ & 0.085 \\
\hline Non-smoker & $1.549(0.592-4.051)$ & 0.372 \\
\hline Former smoker & $1($ ref $)$ & - \\
\hline \multicolumn{3}{|c|}{ Duration of diagnosed type 2 diabetes } \\
\hline Newly diagnosed & $0.261(0.088-0.771)$ & 0.015 \\
\hline$<5$ years & $0.368(0.148-0.912)$ & 0.031 \\
\hline $5-10$ years & $0.248(0.106-0.579)$ & 0.001 \\
\hline $10-15$ years & $0.226(0.096-0.534)$ & 0.001 \\
\hline$>15$ years & $1(\mathrm{ref})$ & - \\
\hline \multicolumn{3}{|l|}{ Family history of diabetes } \\
\hline Yes & $3.465(1.073-11.189)$ & 0.038 \\
\hline No & $3.248(0.946-11.153)$ & 0.061 \\
\hline Unsure & 1 (ref) & - \\
\hline \multicolumn{3}{|l|}{ Diabetes pharmacotherapy } \\
\hline Oral antidiabetic drugs alone & $1.985(1.236-3.188)$ & 0.005 \\
\hline
\end{tabular}




\begin{tabular}{lcc}
\hline \multicolumn{1}{c}{ Characteristic } & \multicolumn{2}{c}{ T\&CM use for type 2 diabetes } \\
OR (95\% CI) & $\boldsymbol{P}$ value \\
\hline Insulin alone & $1.631(0.812-3.273)$ & 0.169 \\
Combination of oral hypoglycemic drugs and & $1(\mathrm{ref})$ & - \\
insulin & & 0.031 \\
Diabetes complications & & - \\
Yes & $0.600(0.377-0.955)$ & 0.021 \\
No & $1(\mathrm{ref})$ & - \\
Concomitant chronic disease & & \\
Yes & $2.949(1.175-7.403)$ & $1(\mathrm{ref})$ \\
No & & \\
\hline
\end{tabular}

The model used was adjusted for age, gender, marital status, employment status, smoking status, duration of diagnosis of type 2 diabetes, family history of diabetes, pharmacotherapy received, presence of diabetes complications, and coexisting chronic disease. The results were based on $n=476$ participants with complete data for all covariates (no missing data).

\section{Discussion}

This study showed T\&CM was commonly used among patients with type 2 diabetes in multi-ethnic communities (Figure 1). Of clinical concern, these patients have a high risk of adverse drug events, principally those of older age and have multiple chronic medical conditions $^{[12]}$. The risk is further increased in light of the challenges of determining specific ingredients in T\&CM products, no tablet size and shape requirements, and lack of postmarketing surveillance ${ }^{[13]}$.

Notably, the current study identified a popular use of herbal medicine. Such biologically-based T\&CM may influence glucose metabolism and interfere with glycemic control $^{[14,15]}$. Reflecting on particular types of herbal remedies consumed by the study participants, bitter melon elicits insulin-mimetic activity, decreases hepatic gluconeogenesis, and is contraindicated in glucose-6-phosphate dehydrogenase deficiency. Garlic increases insulin secretion and potentially interacts with anticoagulant or antiplatelet medications due to its blood thinning effects. Likewise, ginseng causes hypoglycemia by altering hepatic glucose metabolism, interacts with anticoagulant or antiplatelet medications, and causes insomnia, hypertension, and estrogenic effects. Fenugreek decreases carbohydrate absorption, increases insulin secretion, and can cause intestinal gas, bloating, or diarrhea ${ }^{[1]}$. Thus far, most T\&CM studies have found conflicting clinical data and lacked sufficient quality to make a recommendation regarding the efficacy and safety of T\&CM for patients with type 2 diabetes ${ }^{[16,17]}$. Given the high proportion of patients with type 2 diabetes $(61.8 \%)$ reporting utilization of $\mathrm{T} \& \mathrm{CM}$ in this representative sample of a state, the potential effects of T\&CM use on glycemic control, other cardiometabolic risk factors, and health-related quality of life merit further investigation. 


\section{Traditional and complementary medicine use in patients with type 2 diabetes}

Although evidence from existing literature suggests patients with diabetes are more likely to use traditional and
complementary medicine (T\&CM) compared to individuals in the general population, less is known about the
prevalence and pattern of use within urban, multi-ethnic patient communities with type 2 diabetes.

Study Design

\section{$45 \%$ men}

$41 \%$ aged over 60 years

$50 \%$ Malay ethnicity in Petaling district, Malaysia for type 2 diabetes treatment

Knowledge, Attitude \& Perception

1. $30 \%$ believed that T\&CM was safe and effective for diabetes.

2. $80 \%$ would follow a doctor's instructions if the doctor asked them not to use T\&CM.

3. $70 \%$ would discuss T\&CM use with their primary care physician before taking it.

4. $55 \%$ used T\&CM and prescription medication together.

5. $33 \%$ would continue to utilize T\&CM for the long term.

Reasons For T\&CM Use

1. $42 \%$ believed that T\&CM was effective in diabetes control.

2. $10 \%$ cited that T\&CM was easily available and cheaper.

3. $10 \%$ believed that T\&CM had fewer side effects than conventional medicine.

$\checkmark$ Older age, unemployment, oral antidiabetic monotherapy, family history of diabetes, and coexisting chronic illness were associated with higher odds of T\&CM use.

\section{Conclusion}

A high proportion of patients with type 2 diabetes living in urban, multi-ethnic communities reported T\&CM use. The safety and efficacy of T\&CM modalities, as well as the role of T\&CM in conjunction with or as a supplement to conventional means of diabetes care warrant further large-scale investigation.

Figure 1. Research summary of traditional and complementary medicine use in patients with type 2 diabetes in Selangor, Malaysia. 
Because only adults with type 2 diabetes were recruited in our study, the cohort of patients consisted primarily of Malays (49.4\%), Chinese (18.3\%), and Indians (31.7\%). This ethnic composition appeared quite consistent with the findings in the Malaysian National Health and Morbidity Survey 2019, which indicated that Indians had the highest prevalence of a known diagnosis of diabetes (18.5\%), followed by Malays (11.0\%), and Chinese $(8.5 \%)^{[10]}$. The widespread utilization and public interest in T\&CM among a multi-ethnic population could be ascribable to ancestral systems of healing, religious practices, and cultural and spiritual beliefs ${ }^{[7]}$. Patients have preferences for their own treatment decisions and health outcomes, and thus, they may opt to try complementary therapies, albeit they lack appropriate information and understanding. Of note, data from our research suggested that use of T\&CM was not strongly correlated with the lack of confidence towards prescription medications and long appointment intervals to see a doctor, both of which were typically considered by clinicians to be the key factors. This finding stood in contrast to the common assumption and existing research that patient dissatisfaction with waiting time and treatment at a primary care clinic increased the likelihood of T\&CM use ${ }^{[18]}$.

It is important for physicians to enquire whether their patients are using any $\mathrm{T} \& \mathrm{CM}$, especially when they discover unexpectedly poor diabetic control or adverse events. In view of the trust the public has in health care professionals, clinicians should remain neutral about T\&CM use and search for the published medical literature to evaluate the rationale and applicability of T\&CM interventions for specific patients' complaints. For T\&CM modalities that are unsafe or ineffective, dissuading patients is the most reasonable approach. Patients administering herbal remedies or nutritional supplements concurrently with antidiabetic medications require regular primary care follow-up in order to ascertain potential drug interactions attributable to T\&CM use. Health care providers should emphasize the cost of T\&CM therapy to patients, particularly when the efficacy is questionable. Physicians may prescribe vitamins or a diet and recommend exercise for general well-being and specific diagnoses. It is not uncommon that simple home remedies to address minor complaints, for instance, tea for cough and chicken soup for upper respiratory flu are favored by some patients, but it is reasonable to avoid potentially expensive or unnecessary T\&CM practices $^{[19]}$. Before starting any T\&CM, patients should always discuss with their doctor about the possible harmful effects and whether the T\&CM interacts with other medications or with any diseases that the patients have. In particular, if patients are older adults and have impaired renal or hepatic functions, elimination of biologically-based T\&CM from the body would be reduced, thereby resulting in a higher risk of toxicity. As for acupuncture and related T\&CM techniques, currently available evidence has been scarce and varying with 
respect to their efficacy and safety in patients with type 2 diabetes ${ }^{[20]}$. Patients should also be informed about the importance of checking whether their T\&CM practitioners are appropriately qualified and registered under the government regulatory council to ensure the quality and safety of the complementary practices sought by them ${ }^{[21]}$.

\section{Conclusions}

This study demonstrated a high prevalence of T\&CM use among a multi-ethnic patient population with type 2 diabetes. The clinical and sociodemographic characteristics associated with increased T\&CM use comprised age, employment status, oral antidiabetic monotherapy, family history of diabetes, and presence of comorbid chronic condition. Most patients utilized T\&CM to complement conventional medical care because they believed it would help control blood glucose levels. The extensive use of T\&CM, which was reported by $61.8 \%$ of patients, might pose pervasive threats to health. The findings would help pharmacists and clinicians to target interventions to reduce the risk of adverse events associated with the concomitant use of T\&CM in diabetes. Future observational studies will be needed to evaluate the potential interactions, adverse effects, and clinical outcomes in diabetic patients using T\&CM remedies alongside conventional medical treatments.

Author Contributions: Yieng Yii Wong, Azizul Hakim bin Sulaiman, and Anis Aeida binti Mat Jaya researched literature and conceived the study. They were also involved in developing the research proposal and the proforma questionnaire to be used for data collection. Yieng Yii Wong, Azizul Hakim bin Sulaiman, Anis Aeida binti Mat Jaya, Pei Zan Wan, Anchaya A/P Eh Wan, Nurieshah Hanim binti Mohd Anuar, and Rajkumar $\mathrm{A} / \mathrm{L}$ Selvaraju recruited all the patients, collected all the data, and performed data interpretation, analysis, statistical methods, and computation of the results in this study. Yieng Yii Wong wrote the first draft of the manuscript. Kok Pim Kua sought ethics approval, supervised the overall conduct of the study, analyzed and interpreted the results, and edited the manuscript. All authors reviewed and approved the final version of the manuscript.

Acknowledgements: The authors would like to thank the Director General of Health Malaysia for his permission to publish this article. The authors also gratefully acknowledge the Petaling District Health Director, Petaling District Chief Pharmacist, and heads of respective health clinics for their invaluable guidance, encouragement, and support during this research.

Conflicts of Interest: The authors declare that there is no conflict of interest.

\section{References}

1. Birdee GS, Yeh G. Complementary and alternative medicine therapies for diabetes: A clinical review. Clin Diabetes 2010; 28(4): 147-155.

2. Harris PE, Cooper KL, Relton C, et al. Prevalence of complementary and alternative medicine (CAM) use by the general population: A systematic review and update. Int J Clin Pract 2012; 66(10): 924-939.

3. Institute for Public Health (IPH), National Institutes of Health, Ministry of Health Malaysia. 2015. National Health and Morbidity Survey 2015 (NHMS 2015). Vol. IV: Traditional \& Complementary Medicine. 
4. Jou J, Johnson PJ. Nondisclosure of complementary and alternative medicine use to primary care physicians: Findings from the 2012 National Health Interview Survey. JAMA Intern Med 2016; 176(4): $545-546$.

5. Chatterjee S, Khunti K, Davies MJ. Type 2 diabetes. Lancet 2017; 389(10085): 2239-2251.

6. Egede LE, Ye X, Zheng D, et al. The prevalence and pattern of complementary and alternative medicine use in individuals with diabetes. Diabetes Care 2002; 25(2): 324-329.

7. Seet V, Abdin E, Vaingankar JA, et al. The use of complementary and alternative medicine in a multiethnic Asian population: Results from the 2016 Singapore Mental Health Study. BMC Complement Med Ther 2020; 20(1): 52 .

8. Al-Eidi S, Tayel S, Al-Slail F, et al. Knowledge, attitude and practice of patients with type 2 diabetes mellitus towards complementary and alternative medicine. J Integr Med 2016; 14(3): 187-196.

9. Ching SM, Zakaria ZA, Paimin F, et al. Complementary alternative medicine use among patients with type 2 diabetes mellitus in the primary care setting: A cross-sectional study in Malaysia. BMC Complement Altern Med 2013; 13: 148.

10. Institute for Public Health (IPH), National Institutes of Health, Ministry of Health Malaysia. 2020. National Health and Morbidity Survey (NHMS 2019). Vol. I: NCDs-Non-communicable diseases: Risk factors and other health problems.

11. Raosoft, Inc. 2004. Sample size calculator. [Online] Available at: http://www.raosoft.com/samplesize.html [Accessed May 31, 2020].

12. Tan CSS, Lee SWH. Warfarin and food, herbal or dietary supplement interactions: A systematic review. Br J Clin Pharmacol 2021; 87(2): 352-374.

13. Geller AI, Shehab N, Weidle NJ, et al. Emergency department visits for adverse events related to dietary supplements. N Engl J Med 2015; 373(16): 1531-1540.

14. Steyn M, Couchman L, Coombes G, et al. A herbal treatment for type 2 diabetes adulterated with undisclosed drugs. Lancet 2018; 391(10138): 2411.

15. Miller LG. Herbal medicinals: Selected clinical considerations focusing on known or potential drug-herb interactions. Arch Intern Med 1998; 158(20): 2200-2211.

16. Lindquist R, Hoogwerf B. Evidence base for the use of selected complementary and alternative therapies by patients with diabetes. Diabetes Spectrum 2009; 22(4): 199-200.

17. Diabetes Canada Clinical Practice Guidelines Expert Committee, Grossman LD, Roscoe R, Shack AR. Complementary and alternative medicine for diabetes. Can J Diabetes 2018; 42(Suppl 1): S154-S161.

18. Lee GBW, Charn TC, Chew ZH, et al. Complementary and alternative medicine use in patients with chronic diseases in primary care is associated with perceived quality of care and cultural beliefs. Fam Pract 2004; 21(6): 654-660.

19. Rhee SM, Garg VK, Hershey CO. Use of complementary and alternative medicines by ambulatory patients. Arch Intern Med 2004; 164(9): 1004-1009.

20. Liu M, Chen J, Ren Q, et al. Acupuncture and related techniques for type 2 diabetes mellitus: A systematic review protocol. Medicine (Baltimore) 2019; 98(2): e14059.

21. Kim YJ. The current studies of education for a traditional and complementary medicine in Malaysia. $J$ Evid Based Complementary Altern Med 2017; 22(4): 531-537. 


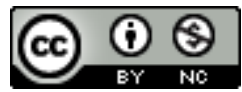

Author(s) shall retain the copyright of their work and grant the Journal/Publisher right for the first publication with the work simultaneously licensed under:

Creative Commons Attribution-NonCommercial 4.0 International (CC BY-NC 4.0). This license allows for the copying, distribution and transmission of the work, provided the correct attribution of the original creator is stated. Adaptation and remixing are also permitted. 